\title{
ON THE FINITE FOURIER TRANSFORMS OF FUNCTIONS WITH INFINITE DISCONTINUITIES
}

\author{
BRANKO SARIC
}

Received 2 April 2001

\begin{abstract}
The introductory part of the paper is provided to give a brief review of the stability theory of a matrix pencil for discrete linear time-invariant singular control systems, based on the causal relationship between Jordan's theorem from the theory of Fourier series and Laurent's theorem from the calculus of residues. The main part is concerned with the theory of the integral transforms, which has proved to be a powerful tool in the control systems theory. On the basis of a newly defined notion of the total value of improper integrals, throughout the main part of the paper, an attempt has been made to present the global theory of the integral transforms, which are slightly more general with respect to the Laplace and Fourier transforms. The paper ends with examples by which the results of the theory are verified.
\end{abstract}

2000 Mathematics Subject Classification: 93C05, 93C55, 32A05, $42 \mathrm{~A} 24$.

1. Introduction. Let $(x, i y) \mapsto f(x, i y)$ be a complex-valued function of the real independent variables $x, y$, and let $\mathscr{D}$ be an arbitrary domain in the $(x, i y)$ plane, where $i$ denotes the imaginary unit. The function $(x, i y) \mapsto f(x, i y)$ is said to be integrable over $\mathscr{D}$, of bounded variation in $\mathscr{D}$ and so forth, with respect to one of the variables, if its real and imaginary parts separately possess those properties for any fixed value of the other variable in $\mathscr{D}$. Accordingly, as it is well known on the basis of Jordan's theorem, if the complex function $s \mapsto f(s)$ of the complex variable $s=x+i y$ is of bounded variation with respect to $i y$ in an arbitrary domain $\mathscr{D}_{s}$ of the $s$-plane: $\mathscr{D}_{s}=\{s: \operatorname{Im} s \in$ $\left[y_{0}, y_{1}\right]$, $\left.\operatorname{Re} s \in\left[x_{0}, x_{1}\right]\right\}\left(0 \in \mathscr{D}_{s}\right)$, then the Fourier trigonometric series of the function

$$
F(s)= \begin{cases}f(s), & \text { if } \operatorname{Im} s \in\left[y_{0}, y_{1}\right], \\ 0, & \text { otherwise, }\end{cases}
$$

at any point $i y$ lying in the closed interval $\left[i y_{0}, i y_{1}\right]$ of the imaginary axis, converges to the function $\mathscr{F}(s)$ (see [8]),

$$
\mathscr{F}(i y)=\operatorname{vp} \sum_{k=-\infty}^{+\infty} \varphi(k) e^{(2 k \pi / a) i y},
$$

where, just as in the integral case, vp denotes the Cauchy principal value of an infinite series, in fact it denotes an infinite series sum in the Cauchy sense; $a \in \mathbb{R}_{+}^{1}$ ( $\mathbb{R}_{+}^{1}$ is a set of points of the positive real axis); and

$$
\varphi(k)=\frac{1}{a i} \int_{i y_{0}}^{i y_{1}} f(s) e^{-(2 k \pi / a) s} d s .
$$


For any $i y \in\left(i y_{1}-i a, i y_{0}+i a\right)$,

$$
\mathscr{F}(i y)=\frac{1}{2}\left[\lim _{\varepsilon \rightarrow 0^{+}} f(i y+i \varepsilon)+\lim _{\eta \rightarrow 0^{+}} f(i y-i \eta)\right],
$$

while at the extreme points of the segment $\left[i y_{1}-i a, i y_{0}+i a\right]$, at which a function iy $\mapsto f(i y)$ is continuous on the right and left, respectively, we have

$$
\mathscr{F}(i y)=\frac{1}{2}\left[\lim _{\varepsilon \rightarrow 0^{+}} f\left(i y_{1}-i a+i \varepsilon\right)+\lim _{\eta \rightarrow 0^{+}} f\left(i y_{0}+i a-i \eta\right)\right] .
$$

Either by the consequence of Bessel's inequality or by the result of the RiemannLebesgue theorem [7], it follows immediately that

$$
\lim _{k \rightarrow \pm \infty} \varphi(k)=0
$$

On the basis of the well-known features of a conform map $z-z_{0}=e^{s}$, (see $[3,9]$ ), if the function $s \mapsto f(s)$ is of period $2 \pi i$ with respect to $i y$, in other words, if the function $z \mapsto \tilde{f}(z): \tilde{f}(z)=f\left[\log \left(z-z_{0}\right)\right]$ is uniform with respect to $i \arg z$ in the $z$-plane, then for $a=2 \pi$ and $-y_{0}=y_{1}=\pi$, (1.3) is transformed into

$$
\varphi(k)=\frac{1}{2 \pi i} \int_{G}^{\cup} \frac{\tilde{f}(z)}{\left(z-z_{0}\right)^{k+1}} d z,
$$

where the symbol $\int_{G}^{\cup}$ denotes an integration over the closed contour $G$ of integration: $G=\left\{z:\left|z-z_{0}\right|=1\right\}$, in this case, in the positive mathematical direction.

On the one hand, since $\lim _{k \rightarrow \pm \infty} \varphi(k)=0$ and

$$
\operatorname{vp} \sum_{k=-\infty}^{+\infty} \varphi(k) e^{s k}=\mathrm{vp} \sum_{k=-\infty}^{-1} \varphi(k) e^{s k}+\mathrm{vp} \sum_{k=0}^{+\infty} \varphi(k) e^{s k},
$$

then both series on the right-hand side of the preceding equation have common region of convergence $\mathscr{R}_{s}=\left\{s: \operatorname{Im} s \in\left[y_{0}, y_{1}\right]\right.$, Re $\left.s \in\left[\tilde{x}_{0}, \tilde{x}_{1}\right]\right\}$, so that for any $c \in\left[\tilde{x}_{0}, \tilde{x}_{1}\right]$, the following holds:

$$
\mathrm{vp} \sum_{k=-\infty}^{+\infty} \varphi(k) e^{(c+i y) k}=\mathscr{F}(c+i y),
$$

where, by (1.3),

$$
\varphi(k)=\frac{e^{-c k}}{2 \pi i} \int_{-i \pi}^{i \pi} f(c+s) e^{-k s} d s=\frac{1}{2 \pi i} \int_{c-i \pi}^{c+i \pi} f(s) e^{-k s} d s,
$$

that is,

$$
\varphi(k)=\frac{1}{2 \pi i} \int_{G_{c}}^{\cup} \frac{\tilde{f}(z)}{\left(z-z_{0}\right)^{k+1}} d z,
$$

where $G_{c}=\left\{z:\left|z-z_{0}\right|=e^{c}\right\}$.

On the other hand, according to Jordan's theorem, for any $c \in\left[x_{0}, x_{1}\right]$, the following holds:

$$
\operatorname{vp} \sum_{k=-\infty}^{+\infty} \varphi_{c}(k) e^{i y k}=\mathscr{F}_{(}(c+i y)
$$


where

$$
\begin{aligned}
\varphi_{c}(k) & =\frac{1}{2 \pi i} \int_{-i \pi}^{i \pi} f(c+s) e^{-k s} d s \\
& =\frac{e^{c k}}{2 \pi i} \int_{c-i \pi}^{c+i \pi} f(s) e^{-k s} d s \\
& =\varphi(k) e^{c k},
\end{aligned}
$$

so that $\mathscr{D}_{S} \subseteq \mathscr{R}_{S}$.

Accordingly, as an immediate consequence of Jordan's theorem, which is slightly more general with respect to the well-known Dirichlet theorem on the expansion of real periodic functions into Fourier trigonometric series [7], the following theorem can be formulated.

THEOREM 1.1. Let the complex-valued function $z \mapsto F(z)$ of the complex variable $z$ be a uniform function of bounded variation with respect to $i \arg z$ in a domain $\mathscr{D}_{z}=\{z$ : $\left.\left|z-z_{0}\right| \in(A, B)\right\}$, where $A \geq 0$ and $B \leq+\infty$. Then, for any $z \in \mathscr{D}_{z}$, the following holds:

$$
\operatorname{vp} \sum_{k=-\infty}^{+\infty} \varphi(k)\left(z-z_{0}\right)^{k}=\mathscr{F}(z),
$$

where

$$
\varphi(k)=\frac{1}{2 \pi i} \int_{G_{c}}^{\cup} \frac{F(z)}{\left(z-z_{0}\right)^{k+1}} d z,
$$

where $G_{c}=\left\{z:\left|z-z_{0}\right|=C, C \in(A, B)\right\}$ and

$$
\mathscr{F}(z)=\frac{1}{2}\left[\lim _{\varepsilon \rightarrow 0^{+}} F\left(z e^{i \varepsilon}\right)+\lim _{\eta \rightarrow 0^{+}} F\left(z e^{-i \eta}\right)\right] .
$$

If $z \mapsto F(z)$ is a uniform regular-analytic function in the disc $0<\left|z-z_{0}\right|<R$, $1<R \leq+\infty$, then by Laurent's theorem, with respect to which the above formulated theorem is slightly more general, for any $z: 0<\left|z-z_{0}\right|<R$, Laurent's expansion $\operatorname{vp} \sum_{k=-\infty}^{+\infty} \varphi(k)\left(z-z_{0}\right)^{k}$ of the function $F(z)$, where $\varphi(k)=(1 / 2 \pi i) \int_{G}^{\cup}(F(z) /(z-$ $\left.\left.z_{0}\right)^{k+1}\right) d z$ and $G=\left\{z:\left|z-z_{0}\right|=C, C \in(0, R)\right\}$, is unique and converges to the value of the function $F(z)$ at that point $z$. Clearly, in this case too, $\lim _{k \rightarrow \pm \infty} \varphi(k)=0$. If, in addition to the above condition, the function satisfies the following condition: $\lim _{z \rightarrow z_{0}}\left(z-z_{0}\right) F(z)=0$, then from Cauchy's calculus of residues it follows, for each $k<0$, that $\varphi(k)=0$ while for $k \geq 0$,

$$
\varphi(k)=\operatorname{Res}_{z=z_{0}} \frac{F(z)}{\left(z-z_{0}\right)^{k+1}}=\frac{F^{(k)}\left(z_{0}\right)}{k !},
$$

where the symbol ! denotes factorial $(0 !=1)$, and $F^{(k)}\left(z_{0}\right)$ is the $k$ th derivative of 
the function $F(z)$ at the point $z_{0}$, that is, for each $k \geq k_{\infty}$, where $k_{\infty}$ is the smallest nonnegative integer such that $\lim _{|z| \rightarrow+\infty}\left(z F(z) /\left(z-z_{0}\right)^{1+k_{\infty}}\right)=0$,

$$
\varphi(k)=-\sum_{\kappa=1}^{n} \operatorname{Res}_{z=z_{\kappa}} \frac{F(z)}{\left(z-z_{0}\right)^{k+1}},
$$

considering the fact that all points of infinite discontinuity (all singularities) $z_{\kappa}$ ( $\kappa=$ $1,2, \ldots, n)$ of the function $F(z)$ are outside the region of convergence $0<\left|z-z_{0}\right|<R$.

Now, Laurent's expansion, representing for $|z|=1$ the discrete finite Fourier transform, can be said to be analogous to the so-called $Z$-transform having practical application in discrete control systems theory. Thus, for a class of discrete linear timeinvariant singular control systems, the matrix sequence $\Phi(k)$ in Laurent's expansion of the matrix function $z \mapsto z^{-1} \mathbf{P}\left(z^{-1} \mathbf{P}-\mathbf{A}\right)^{-1}: \sum_{k=0}^{+\infty} \Phi(k) z^{k}$, whenever it exists-where $n \times n$ matrix $\mathbf{A}$ and singular $n \times n$ matrix $\mathbf{P}$ are, in the general case, constant matrices defined on the field of the complex numbers-is a solution for the matrix difference equation $\triangle \Phi(k) \mathbf{P}=\Phi(k)(\mathbf{A}-\mathbf{P}) ; k \geq 0$, with initial condition $\Phi(0) \mathbf{P}=\mathbf{P}$. This form of Laurent's expansion exists if and only if $\lim _{|z| \rightarrow+\infty} \mathbf{P}(z \mathbf{P}-\mathbf{A})^{-1}=\mathbf{0}$. Following the results presented by Lewis and Mertzios in [4, 5] (taken over from [2]), it can be easily shown that if ind $\hat{\mathbf{P}}=1$, more exactly if $\operatorname{rank} \hat{\mathbf{P}}=\operatorname{rank} \hat{\mathbf{P}}^{2}$, where the matrix $\hat{\mathbf{P}}^{D}$ is a Drazin inverse matrix of the singular matrix $\hat{\mathbf{P}}: \hat{\mathbf{P}}=\mathbf{P}(\nu \mathbf{P}-\mathbf{A})^{-1}$ (clearly under the condition that there exists a constant $v$ such that the matrix $(\nu \mathbf{P}-\mathbf{A})$ is invertible), then $\Phi(k)=\hat{\mathbf{P}}^{D}\left(\hat{\mathbf{P}}^{D} \hat{\mathbf{A}}\right)^{k} \hat{\mathbf{P}}, k \geq 0$ and $\Phi(k)=\mathbf{0}, k<0$. In addition, if $\operatorname{det} \mathbf{A} \neq 0$ then $\sum_{\kappa=1}^{\operatorname{rank} \mathbf{P}} \operatorname{Res}_{z=z_{\kappa}}\left(\mathbf{P}(z \mathbf{P}-\mathbf{A})^{-1} / z\right)=\mathbf{P A}^{-1}$, where $z_{\kappa} s$ are singularities of the function $z \mapsto \mathbf{P}(z \mathbf{P}-\mathbf{A})^{-1}$. If the radius of convergence of the power series $\sum_{k=0}^{+\infty} \Phi(k) z^{k}$ is larger than 1, in other words, if all singularities of the function $z \mapsto z^{-1} \mathbf{P}\left(z^{-1} \mathbf{P}-\mathbf{A}\right)^{-1}$ are outside the unit circle, then the matrix pencil $\left(z^{-1} \mathbf{P}-\mathbf{A}\right)$ is asymptotic stable and, by (1.6), $\lim _{k \rightarrow+\infty} \Phi(k)=\mathbf{0}$.

In the case when either the real or imaginary part of a complex-valued function $s \mapsto F(s)$ is not Riemann-integrable over $[-i \pi, i \pi]$, there is practically at least one infinite discontinuity (singularity) of the function $\tilde{F}(z)$ that lies onto the contour of integration $G=\{z:|z|=1\}$. This leads to a basic question: is there a theory of discrete Fourier transform in this global case too? In other words, is there a global theorem of discrete Fourier transform by which we can prove that an infinite Laurent's power series on the left-hand side of (1.14) is summable, and for which an inversion formula is in the form of (1.15)? Clearly, it would be desirable to draw a conceptual distinction between a summation of series in the Cauchy sense and its summability [7]. An infinite series, which is either indefinite divergent or convergent in the Cauchy sense, and to which its sum can be uniquely joined, one way or another, is said to be summable.

An answer to the former questions will be given in what follows.

\section{Main results}

2.1. The total value of an improper integral. It may be assumed, without loss of generality, that a uniform analytic function $z \mapsto f(z)$ has only one isolated singularity at the point $z_{0}$ lying onto an arbitrary contour of integration $\Gamma$. Then, the total value vt 
of an improper integral of $f(z)$ over $\Gamma$ is defined to be the sum of the Cauchy principal value vp and the Jordan singular value vs

$$
\mathrm{vt} \int_{\Gamma} f(z) d z=\mathrm{vp} \int_{\Gamma} f(z) d z+\mathrm{vs} \int_{\Gamma} f(z) d z .
$$

On the other hand, the Jordan singular value vs is defined to be equal to the limiting values, as $\varepsilon \rightarrow 0^{+}$, of the integrals of function $f(z)$ along both arc parts of a circular path of integration $\gamma_{\varepsilon}$ by which the singularity of $f(z)$ at the point $z_{0}$ is surrounded:

$$
\text { vs } \int_{\Gamma} f(z) d z=\lim _{\varepsilon \rightarrow 0^{+}} \int_{\gamma_{\varepsilon}}^{\hat{P Q}} f(z) d z+\left\{\begin{array}{l}
0 \\
2 \pi i \operatorname{Res}_{z=z_{0}} f(z),
\end{array}\right.
$$

where the points $P$ and $Q$ are the intersection points of the circular contour $\gamma_{\varepsilon}$ and the integration contour $\Gamma$. By the calculus of residues (cf. [9, Equation (2) of Subsection 3.6.4, page 233]), if the singularity of $f(z)$ at the point $z_{0}$ is a simple pole, then

$$
\text { vs } \int_{\Gamma} f(z) d z=-\alpha i \operatorname{Res}_{z=z_{0}} f(z)+\left\{\begin{array}{l}
0 \\
2 \pi i \operatorname{Res}_{z=z_{0}} f(z),
\end{array}\right.
$$

where $\alpha$ is the limit, as the points $P$ and $Q$, along the path of integration $\Gamma$, tends to the point $z_{0}$, of the angle of tangents to $\Gamma$ at those points. In the general case, if a uniform analytic function $z \mapsto f(z)$ has, onto and inside an arbitrary closed contour of integration $\Gamma$, only isolated singularities $z_{\kappa} \mathrm{s}(\kappa=0,1,2, \ldots, m$ and $\kappa=m+1, m+$ $2, \ldots, m+n$, respectively, the number of isolated singularities of the function must be finite, because in the opposite case there exists a point of accumulation which is not an isolated singularity) then by Cauchy's fundamental theorem on residues and by (2.1) and (2.2), it follows immediately that

$$
\mathrm{vt} \int_{\Gamma}^{\cup} f(z) d z=2 \pi i\left\{\begin{array}{l}
\sum_{\kappa=0}^{m+n} \operatorname{Res}_{z=z_{\kappa}} f(z) \\
\sum_{k=m+1}^{m+n} \operatorname{Res}_{z=z_{\kappa}} f(z) .
\end{array}\right.
$$

2.2. The finite Fourier transform. If in a domain $\mathscr{D}_{s}$ of $s$-plane-defined at the beginning of the introductory part of this paper, but now with the changeable upper limit: $\operatorname{Im} s \in\left[y_{0}, y\right]$ 一the complex-valued function $s \mapsto F(s)$ of the complex variable $s$ is a uniform analytic function having an infinite discontinuity (singularity) at the point $s_{0}=0$, then it follows immediately from (2.4) that

$$
\frac{1}{2 \pi i} \mathrm{vt} \int_{\Gamma}^{\cup} F(s) e^{w(i y-s)} d s=\left\{\begin{array}{l}
\sum_{\kappa=0}^{n} \operatorname{Res}_{s=s_{K}}\left[F(s) e^{w(i y-s)}\right], \\
\sum_{\kappa=1}^{n} \operatorname{Res}_{s=s_{K}}\left[F(s) e^{w(i y-s)}\right],
\end{array}\right.
$$


where $w$ is a complex parameter, and the contour of integration $\Gamma$ consists of the imaginary axis segment $\left[i y_{0}, i y\right]$ and an arbitrary Jordan's curve $\gamma\left(\gamma \in \mathscr{D}_{s}\right)$ connecting the points $i y_{0}$ and $i y$, while $s_{\kappa} s(\kappa=1,2, \ldots, n)$ are isolated points of infinite discontinuity of $F(s)$ within $\Gamma$. Therefore, the following holds:

$$
\frac{1}{2 \pi i} \mathrm{vt} \int_{i y_{0}}^{i y} F(s) e^{w(i y-s)} d s=\left\{\begin{array}{l}
f_{\gamma}(w, i y) e^{w i y}-\sum_{\kappa=0}^{n} \operatorname{Res}_{s=s_{K}}\left[F(s) e^{w(i y-s)}\right] \\
f_{\gamma}(w, i y) e^{w i y}-\sum_{\kappa=1}^{n} \operatorname{Res}_{s=s_{K}}\left[F(s) e^{w(i y-s)}\right]
\end{array}\right.
$$

where $f_{\gamma}(w, i y)=(1 / 2 \pi i) \int_{\gamma}^{i y i y_{0}} F(s) e^{-s w} d s$.

Let, further, the segment Res $\in\left[x_{0}, x_{1}\right]$ of lines, which are parallel to the real axis and are distant $i y$ and $i y_{0}$ from it, be a set of points of finite discontinuity of $F(s)$. Then, the function $s \mapsto \tilde{F}(s)$ can be defined on the domain $\operatorname{Re} s \in\left[x_{0}, x_{1}\right]$ of $s$-plane, as follows:

$$
\tilde{F}(s)= \begin{cases}F(s), & \text { if } \operatorname{Im} s \in\left(y_{0}, y\right), \\ f(s), & \text { otherwise, }\end{cases}
$$

where $f(s)$ is a function such that, for $\operatorname{Re} s \in\left[x_{0}, x_{1}\right]$ and $\operatorname{Im} s \geq y$,

$$
f(s)=\lim _{\varepsilon \rightarrow 0^{+}} F[\operatorname{Re} s+i(y-\varepsilon)]
$$

and, for $\operatorname{Im} s \leq y_{0}$,

$$
f(s)=\lim _{\eta \rightarrow 0^{+}} F\left[\operatorname{Re} s+i\left(y_{0}+\eta\right)\right]
$$

In the half-plane for $\operatorname{Re} s \leq x_{0}, \tilde{F}(s)=\tilde{F}\left(\operatorname{Re} x_{0}+i \operatorname{Im} s\right)$, while for $\operatorname{Re} s \geq x_{1}, \tilde{F}(s)=$ $\tilde{F}\left(\operatorname{Re} x_{1}+i \operatorname{Im} s\right)$.

Further, we will consider a bilinear map $z=e^{2 i \arctan (-i s)}=(1+s) /(1-s)$, which is proved as follows. On the one hand,

$$
\begin{aligned}
e^{2 i \arctan (-i s)} & =\cos [2 \arctan (-i s)]+i \sin [2 \arctan (-i s)] \\
& =[1+i \tan [2 \arctan (-i s)]] \cos [[2 \arctan (-i s)]] \\
& =[1+i \tan [\arctan (-i s)]]^{2} \cos ^{2}[\arctan (-i s)] \\
& =(1+s)^{2} \cos ^{2}[\arctan (-i s)] .
\end{aligned}
$$

And on the other, $\cos ^{2}[\arctan (-i s)]=1-\sin ^{2}[\arctan (-i s)]$; more exactly,

$$
\cos ^{2}[\arctan (-i s)]\left[1+\tan ^{2}[\arctan (-i s)]\right]=\cos ^{2}[\arctan (-i s)]\left(1-s^{2}\right)=1 .
$$


From the preceding equalities, it follows that $e^{2 i \arctan (-i s)}=(1+s)^{2} /\left(1-s^{2}\right)=$ $(1+s) /(1-s)$. Resuming: considering the well-known properties of a bilinear map $z=e^{2 i \arctan (-i s)}=(1+s) /(1-s)$, for every $w$,

$$
\begin{aligned}
\mathrm{vt} \int_{-i \infty}^{+i \infty} e^{-s w} d s & =\mathrm{vt} \int_{G}^{\cup} \frac{2 e^{-w((z-1) /(z+1))}}{(z+1)^{2}} d z \\
& =2 \mathrm{vt} \int_{G}^{\cup} \frac{\sum_{k=0}^{+\infty}\left((-w)^{k} / k !\right)(1-2 /(z+1))^{k}}{(z+1)^{2}} d z=0,
\end{aligned}
$$

where $G$ is a unit circle in the $z$-plane. Accordingly,

$$
\mathrm{vt} \int_{\mathscr{I}}^{i y\left(\tilde{\mp} \hat{i \infty)} i y_{0}\right.} e^{w(i y-s)} d s=\mathrm{vt} \int_{\mathscr{I}}^{i y 0 i y_{0}} e^{w(i y-s)} d s=\frac{1}{w}\left[e^{w\left(i y-i y_{0}\right)}-1\right]
$$

where $\int_{\mathscr{I}}^{i y(\tilde{\mp} \hat{i \infty}) i y_{0}}$ denotes an integration over the expanded imaginary axis part connecting the points $i y_{0}$ and $i y$, via infinity.

Let $F(s)=i c e^{i a(i y-s)}$ for $s \in \mathscr{D}_{s}$, where $c$ and $a$ are real constants, $a$ being positive. Then, according to (2.13), if the function $s \mapsto \tilde{F}(s)$ defined by (2.7)—which can be said, in this acute case, to be continuous-satisfies the condition

$$
\mathrm{vt} \int_{-i \infty}^{+i \infty} \tilde{F}(s) e^{w(i y-s)} d s=0
$$

it follows, for $w \in \mathscr{R}_{w}, \mathscr{R}_{w}=\{w: \operatorname{Im} w>-a\}$, that

$$
\mathrm{vt} \int_{\mathscr{g}}^{i y\left(\tilde{\mp} \hat{i \infty)} i y_{0}\right.}(w+i a) \tilde{F}(s) e^{w(i y-s)} d s=i c\left[e^{(w+i a)\left(i y-i y_{0}\right)}-1\right],
$$

that is,

$$
\lim _{|w| \rightarrow+\infty} \mathrm{vt} \int_{\mathscr{I}}^{i y(\tilde{\digamma} \hat{\imath}) i y_{0}}(w+i a) \tilde{F}(s) e^{w(i y-s)} d s=-\tilde{F}(i y) .
$$

In the limit, as $a \rightarrow 0^{+}$, the region $\mathscr{R}_{w}$ is reduced to $\mathscr{R}_{w}=\{w: \operatorname{Im} w \geq 0\}$.

By (2.16), which is independent of the form of the function $s \mapsto F(s)$, if an arbitrary uniform analytic function $s \mapsto F(s)$ has isolated points of infinite discontinuity within the domain $\mathscr{D}_{s}$ and if the following condition is satisfied

$$
\mathrm{vt} \int_{-i \infty}^{+i \infty} \tilde{F}(s) e^{w(i y-s)} d s=2 \pi i\left\{\begin{array}{l}
\sum_{\kappa=0}^{m+n} \operatorname{Res}_{s=s_{\kappa}}\left[F(s) e^{w(i y-s)}\right] \\
\sum_{\kappa=m+1}^{m+n} \operatorname{Res}_{s=s_{K}}\left[F(s) e^{w(i y-s)}\right]
\end{array}\right.
$$


where $s_{\kappa} s(\kappa=0,1, \ldots, m)$ are points of infinite discontinuity of $F(s)$ onto the imaginary axis, while $s_{\kappa} s(\kappa=m+1, m+2, \ldots, m+n)$ are points of infinite discontinuity of $F(s)$ in either part of the domain $\mathscr{D}_{S}$ in which $\operatorname{Re} s<0$ or $\operatorname{Re} s>0$, then, because of the existence of the function residues on the right-hand side of the preceding equation, in this general case, the region $\mathscr{R}_{w}$ of the integral convergence,

$$
\lim _{|w| \rightarrow+\infty} \mathrm{vt} \int_{i y_{0}}^{i y}(w+i a) F(s) e^{w(i y-s)} d s=-\lim _{\varepsilon \rightarrow 0^{+}} F(i y-i \varepsilon),
$$

is $\mathscr{R}_{w}=\{w: \operatorname{Im} w>0\}$.

Clearly, if there are no points of the infinite discontinuity of $F(s)$ either in the part of the domain $\mathscr{D}_{s}$ in which $\operatorname{Re} s \leq 0$ or $\operatorname{Re} s \geq 0$, then the sums of residues on the righthand side of condition (2.17) both vanish; and in that case, condition (2.17) is reduced to condition (2.14), while the region $\mathscr{R}_{w}$ of the preceding integral convergence, is just as in the case of the integral convergence (2.16), that is, $\mathscr{R}_{w}=\{w: \operatorname{Im} w>-a\}$, and in the limit, as $a \rightarrow 0^{+}, \mathscr{R}_{w}=\{w: \operatorname{Im} w \geq 0\}$.

Since the foundation stone of the whole theory is the preceding result, it would be desirable to say a few words about the conditions under which it holds. Namely, condition (2.17) is transformed by a bilinear map into

$$
\mathrm{vt} \int_{G}^{\cup} \tilde{F}\left(\frac{z-1}{z+1}\right) \frac{e^{w((1-z) /(1+z))}}{(z+1)^{2}} d z=2 \pi i\left\{\begin{array}{l}
\sum_{\kappa=0}^{m+n} \operatorname{Res}_{z=z_{\kappa}}\left[F\left(\frac{z-1}{z+1}\right) \frac{e^{w((1-z) /(1+z))}}{(z+1)^{2}}\right] \\
\sum_{k=m+1}^{m+n} \operatorname{Res}_{z=z_{\kappa}}\left[F\left(\frac{z-1}{z+1}\right) \frac{e^{w((1-z) /(1+z))}}{(z+1)^{2}}\right],
\end{array}\right.
$$

where $G$ is a unit circle in the $z$-plane. Considering the fact that the residue at the point $z=-1$ of $\tilde{F}((z-1) /(z+1))$ (which is a continuous function over the arc parts passing through the points $z=e^{2 i \arctan y}$ and $z=-1$, as well as the points $z=e^{2 i \arctan y_{0}}$ and $z=-1$, of the unit circle and circles centered at the line $\operatorname{Re} z=-1$, respectively) depends only upon the behavior of the function $s \mapsto \tilde{F}(s)$ in a neighborhood of a set of points at infinity, on the one hand,

$$
\operatorname{Res}_{z=-1}\left[\tilde{F}\left(\frac{z-1}{z+1}\right) \frac{e^{w((1-z) /(1+z))}}{(z+1)^{2}}\right]=0 \text {; }
$$

and on the other hand, it is easily shown by an application of Cauchy-Goursat's integral theorem that in those circumstances, condition (2.19) is an immediate consequence of (2.4).

To sum up, if $F(s)$ is a uniform analytic function inside $\mathscr{D}_{s}$, the region $\mathscr{R}_{w}=\{w$ : $\operatorname{Im} w>0\}$ is that of the integral convergence

$$
\lim _{|w| \rightarrow+\infty} \mathrm{vt} \int_{0}^{i\left(y-y_{0}\right)}(w+i a) F(i y-s) e^{s w} d s=-\lim _{\varepsilon \rightarrow 0^{+}} F(i y-i \varepsilon)
$$


By the second Jordan's lemma from the calculus of residues, since the integral function $w \mapsto v t \int_{0}^{i\left(y-y_{0}\right)} F(i y-s) e^{s w} d s$ is a regular-analytic function in the whole $w$-plane, it follows, for any $c>0$ (more exactly $c \geq 0$, as pointed out indirectly by Mitrinović's result in [6, page 22]), that

$$
\frac{1}{2 \pi i} \lim _{\omega \rightarrow+\infty} \int_{i c-\omega}^{i c+\omega}\left[\mathrm{vt} \int_{i y_{0}}^{i y} F(s) e^{-s w} d s\right] e^{w i y} d w=\frac{1}{2} \lim _{\varepsilon \rightarrow 0^{+}} F(i y-i \varepsilon) .
$$

Similarly, we can prove that, for any $c<0(c \leq 0)$,

$$
\frac{1}{2 \pi i} \lim _{\omega \rightarrow+\infty} \int_{i c-\omega}^{i c+\omega}\left[\mathrm{vt} \int_{i y}^{i y_{1}} F(s) e^{-s w} d s\right] e^{w i y} d w=\frac{1}{2} \lim _{\eta \rightarrow 0^{+}} F(i y+i \eta) .
$$

Without loss of generality, if the function $s \mapsto F(s)$, which is a uniform analytic function in the whole $s$-plane, has no points of infinite discontinuity in the segment $\left[i y_{0}, i y\right]$, with exception of the point $s_{0}=0$, at which it has a simple pole as singularity, from (2.6) we obtain

$$
\begin{aligned}
\frac{1}{2 \pi i} & \operatorname{vp} \int_{i y_{0}}^{i y} F(s) e^{w(i y-s)} d s \\
& =f_{\gamma}(w, i y) e^{w i y}-\sum_{\kappa=1}^{n} \operatorname{Res}_{s=s_{\kappa}}\left[F(s) e^{w(i y-s)}\right]-\frac{1}{2} \operatorname{Res}_{s=s_{0}}\left[F(s) e^{w(i y-s)}\right] .
\end{aligned}
$$

Following the result of one of Baskin's general theorems [1], we obtain, for every $w$ such that $s F(s) e^{-s w}=O\left(|s|^{-1}\right.$ ) for Re $s<0$, (as pointed out by Mitrinović, see either [8, page 102] or [6, page 22])

$$
\begin{aligned}
\frac{1}{2 \pi i} & \mathrm{vp} \int_{-i \infty}^{+i \infty} F(s) e^{-s w} d s \\
& =\lim _{\substack{y_{0} \rightarrow-\infty \\
y \rightarrow+\infty}} f_{\gamma}(w, i y)-\sum_{\kappa=1}^{n} \operatorname{Res}_{s=s_{\kappa}}\left[F(s) e^{-s w}\right]-\frac{1}{2} \operatorname{Res}_{s=s_{0}}\left[F(s) e^{-s w}\right] \\
& =\sum_{\kappa=n+1}^{m+n} \operatorname{Res}_{s=s_{\kappa}}\left[F(s) e^{-s w}\right]+\frac{1}{2} \operatorname{Res}\left[F(s) e^{-s w}\right],
\end{aligned}
$$

that is,

$$
\lim _{\substack{y_{0 \rightarrow-\infty} \\ y \rightarrow+\infty}} f_{\gamma}(w, i y)=\sum_{\kappa=0}^{m+n} \operatorname{Res}_{s=s_{\kappa}}\left[F(s) e^{-s w}\right]
$$

where $s_{\kappa} s(\kappa=n+1, n+2, \ldots, n+m)$ are points of infinite discontinuity of $F(s)$ in the half-plane $\operatorname{Re} s<0$. According to (2.26), it follows for $\operatorname{Im} w=0$ that if all points of infinite discontinuity of $F(s)$ are in the half-plane $\operatorname{Re} s<c$, where $c$ is such that

$$
\gamma_{c}=\left\{s: s-i y_{0}=r e^{i \arctan \left(y_{0} / c\right)} \cup s-i y=r e^{-i \arctan (y / c)}, r \in[0, c]\right\},
$$


and $F(s)=O\left(|s|^{-1}\right)$ for $\operatorname{Re} s \geq c$, then for $\operatorname{Re} v>c$

$$
\begin{aligned}
\lim _{w \rightarrow+\infty} \int_{-\omega}^{0} \sum_{\kappa=0}^{m+n} \operatorname{Res}_{s=s_{K}}\left[F(s) e^{-s w}\right] e^{w v} d w \\
\quad=\lim _{\omega \rightarrow+\infty} \int_{-\omega}^{0}\left[\lim _{y_{0} \rightarrow-\infty} f_{\gamma_{c}}(w, i y)\right] e^{w v} d w \\
=\frac{1}{2 \pi i} \lim _{\omega \rightarrow+\infty} \int_{-\omega}^{0} \mathrm{vp} \int_{c-i \infty}^{c+i \infty} F(s) e^{w(v-s)} d s d w \\
=\frac{1}{2 \pi i} \mathrm{vp} \int_{c-i \infty}^{c+i \infty} \frac{F(s)}{(v-s)} d s=F(v) .
\end{aligned}
$$

On the other hand, it is also well known on the basis of Jordan's theorem, mentioned at the beginning of this paper, more exactly of the Fourier integral theorem, that the Fourier integral of the complex function $s \mapsto F(s)$ (defined by (1.1) in such a way that now $f(s)$ is of bounded variation with respect to iy in a domain $\mathscr{D}_{s}$ of the $s$-plane with the changeable upper limit $\left.\operatorname{Im} s \in\left[y_{0}, y\right]\right)$ converges for each $i \zeta \in\left[i y_{0}, i y\right]$ to the function $\mathscr{F}(i \zeta)$ defined by an equation similar to (1.4) (see [8]):

$$
\begin{aligned}
\mathscr{F}(i \zeta) & =\frac{1}{\sqrt[2]{2 \pi} i} \mathrm{vp} \int_{-i \infty}^{+i \infty} \phi(-i s, i y) e^{y s} e^{i(i y-i \zeta) s} d s \\
& =\frac{1}{\sqrt[2]{2 \pi}} \mathrm{vp} \int_{-\infty}^{+\infty} \phi(s, i y) e^{i y s} e^{-(i y-i \zeta) s} d s, \\
\phi(\zeta, i y) e^{i y \zeta} & =\frac{1}{\sqrt[2]{2 \pi} i} \int_{i y_{0}}^{i y} f(s) e^{(i y-s) \zeta} d s=\frac{1}{\sqrt[2]{2 \pi}} \int_{y_{0}}^{y} f(i s) e^{(i y-i s) \zeta} d s .
\end{aligned}
$$

On account of the uniform convergence of the power series of the exponential function and properties of a bilinear map, (2.30) is transformed into

$$
\Phi(\zeta, i y)=\phi(\zeta, i y) e^{i y \zeta}=\sum_{k=0}^{+\infty} \varphi(k, i y) \zeta^{k},
$$

where, for $z_{0}=1$ and $z(i y)=(1+i y) /(1-i y)$ as well as $z(i y) \in \hat{G}, \hat{G}$ is an upper semicircle of the unit circle $G$,

$$
\begin{aligned}
\varphi(k, i y) & =\frac{1}{\sqrt[2]{2 \pi}} \frac{1}{i k !} \int_{0}^{i\left(y-y_{0}\right)} s^{k} f(i y-s) d s \\
& =\sqrt[2]{\frac{2}{\pi}} \frac{1}{i k !} \int_{G}^{z(i y) z_{0}} \frac{(z-1)^{k} \tilde{f}[z(i y), z]}{(z+1)^{k+2}} d z .
\end{aligned}
$$

For any real $c$,

$$
\begin{aligned}
\Phi[(\zeta+i c), i y] & =\sum_{k=0}^{+\infty} \varphi(k, i y)(\zeta+i c)^{k}, \\
\mathscr{F}(i y-i \zeta) e^{-\zeta c} & =\frac{1}{\sqrt[2]{2 \pi}} \mathrm{vp} \int_{-\infty}^{+\infty} \phi(s+i c, i y) e^{i y(s+i c)} e^{-i \zeta s} d s
\end{aligned}
$$


that is,

$$
\begin{aligned}
\phi(w, i y) & =\frac{1}{\sqrt[2]{2 \pi} i} \int_{i y_{0}}^{i y} f(s) e^{-w s} d s, \\
\mathscr{F}(i \zeta) & =\frac{1}{\sqrt[2]{2 \pi}} \lim _{\omega \rightarrow+\infty} \int_{i c-\omega}^{i c+\omega} \phi(w, i y) e^{i \zeta w} d w .
\end{aligned}
$$

Similarly, if the integral $\lim _{\substack{y_{0} \rightarrow-\infty \\ y \rightarrow+\infty}} \int_{i y_{0}}^{i y} f(s) e^{-w s} d s$ converges absolutely in the region $\mathscr{R}_{w}, \mathscr{R}_{w}=\{w: a \leq \operatorname{Im} w \leq b, 0 \in[a, b]\}$, then, for any $w \in \mathscr{R}_{w}$, the following holds:

$$
\begin{aligned}
\phi(w) & =\frac{1}{\sqrt[2]{2 \pi} i} \mathrm{vp} \int_{-i \infty}^{+i \infty} f(s) e^{-w s} d s, \\
\mathscr{F}(i \zeta) & =\frac{1}{\sqrt[2]{2 \pi}} \lim _{\omega \rightarrow+\infty} \int_{i c-\omega}^{i c+\omega} \phi(w) e^{i \zeta w} d w .
\end{aligned}
$$

To sum up again, as yet another immediate consequence of Jordan's theorem, more exactly of the integral Fourier theorem, the preceding two systems of the integral equations which, respectively, define the finite Laplace transform and the so-called bilateral Laplace transform reducing for $c=0$ to the Fourier transform, have been just obtained. In addition, the one-sided Laplace transform is defined by (2.28). On the other hand, the equation, obtained by summation of (2.22) and (2.23) for $c=0$, generalizes the Fourier integral theorem. Therefore, previously redefining the wellknown Dirichlet's condition as follows: a periodic complex-valued function $s \mapsto F(s)$ of the complex variable $s$ and of period $2 \pi i$ with respect to $i y$, which is a sectionally analytic function with respect to $i y$, in the sense that it has only a finite number $k$ of a set of points $\operatorname{Im} s=y_{k}$ of finite discontinuity in the linear domain $\mathscr{D}_{s}=\{s: \operatorname{Im} s \in$ $[-\pi, \pi]\}$ of the $s$-plane, can be said to satisfy the global Dirichlet's condition in $\mathscr{D}_{s}$; we are able to establish the fact that (2.21) proves indirectly the global Dirichlet theorem.

THEOREM 2.1. Let the complex-valued function iy $\mapsto F(i y)$ of the real variable $y$ be a periodic function, of period $2 \pi i$ on the imaginary axis. Then, if it satisfies the global Dirichlet condition in the linear domain $\mathscr{D}_{S}: \mathscr{D}_{S}=\{i y: i y \in[-i \pi, i \pi]\}$, for any $i y \in \mathscr{D}_{s}$, and $i y \neq i y_{k}$, where $i y_{k} s$ are points of the infinite discontinuity of $F(i y)$ in $\mathscr{D}_{s}$, the following holds:

$$
\mathrm{vt} \sum_{k=-\infty}^{+\infty} \varphi(k) e^{i y k}=\mathscr{F}(i y)
$$

where

$$
\varphi(k)=\frac{1}{2 \pi i} \mathrm{vt} \int_{-i \pi}^{i \pi} F(s) e^{-k s} d s,
$$

and, for $i y \neq \pm i \pi$ as well as for $i y= \pm i \pi$,

$$
\begin{aligned}
& \mathscr{F}(i y)=\frac{1}{2}\left[\lim _{\varepsilon \rightarrow 0^{+}} F(i y+i \varepsilon)+\lim _{\eta \rightarrow 0^{+}} F(i y-i \eta)\right], \\
& \mathscr{F}(i y)=\frac{1}{2}\left[\lim _{\varepsilon \rightarrow 0^{+}} F(-i \pi+i \varepsilon)+\lim _{\eta \rightarrow 0^{+}} F(i \pi-i \eta)\right],
\end{aligned}
$$

respectively. 
The total value vt of the infinite series on the left-hand side of (2.36), can be said to be defined in the more general sense with respect to the Cauchy principal value vp. In other words, an infinite series, which in the general case indefinitely diverges in the Cauchy sense, is summable and has a defined sum. Clearly, in this case, on the one hand, the result of the Riemann-Lebesgue theorem $\lim _{k \rightarrow \pm \infty} \varphi(k)=0$, which is also a consequence of Bessel's inequality, does not hold, moreover it is possible that $\lim _{k \rightarrow \pm \infty} \varphi(k)= \pm \infty$, and on the other hand, for any $c \in\left[x_{0}, x_{1}\right]$, the following holds:

$$
\mathrm{vt} \sum_{k=-\infty}^{+\infty} \varphi_{c}(k) e^{i y k}=\mathscr{F}(c+i y),
$$

where, by (2.53),

$$
\varphi_{c}(k)=\frac{1}{2 \pi i} \mathrm{vt} \int_{-i \pi}^{i \pi} F(c+s) e^{-k s} d s .
$$

Accordingly, just as at the beginning of the paper, the following theorem can be formulated, as an immediate consequence of the preceding theorem.

THEOREM 2.2. Let the complex-valued function $z \mapsto \tilde{F}(z)$ be a uniform function satisfying the global Dirichlet condition inside a domain $\mathscr{D}_{z}$ of the $z$-plane, $\mathscr{D}_{z}=\{z$ : $\left.\left|z-z_{0}\right| \in(A, B)\right\}$, where $A \geq 0$ and $B \leq+\infty$, in other words, let $\tilde{F}(z)$ be a uniform sectionally analytic function with respect to $i \arg z$ inside $\mathscr{D}_{z}$. Then, for any $z$ such that $z \in G_{c}, G_{c}=\left\{z:\left|z-z_{0}\right|=C, C \in(A, B)\right\}$ and $z \neq z_{k}$, where $z_{k}$ s are points belonging to $G_{c}$ at which $\tilde{F}(z)$ has the infinite discontinuity, of course whenever they exist, the following holds:

$$
\mathrm{vt} \sum_{k=-\infty}^{+\infty} \varphi(k)\left(z-z_{0}\right)^{k}=\tilde{\mathscr{F}}(z)
$$

where

$$
\begin{aligned}
& \varphi(k)=\frac{1}{2 \pi i} \mathrm{vt} \int_{G_{c}}^{\cup} \frac{\tilde{F}(z)}{\left(z-z_{0}\right)^{k+1}} d z, \\
& \tilde{\mathscr{F}}(z)=\frac{1}{2}\left[\lim _{\varepsilon \rightarrow 0^{+}} \tilde{F}\left(z e^{i \varepsilon}\right)+\lim _{\eta \rightarrow 0^{+}} \tilde{F}\left(z e^{-i \eta}\right)\right] .
\end{aligned}
$$

Clearly, the result of the preceding theorem can be said to generalize that of Laurent's theorem, as well as the result of Theorem 1.1.

\subsection{The proof of the global Dirichlet theorem}

2.3.1. Analysis of an idea of the theorem's proof. Without loss of generality, we may assume that the complex-valued function $g(w, i y)$, where the complex variable $i y$ is independent with respect to the complex variable $w$, has infinitely but countable many simple real poles $a_{1}, a_{2}, \ldots$ with the points of accumulations at infinity. In that emphasized case, there exists an infinite sequence of circular contours of integration $\Omega_{r_{n}}$, centered at the origin and of radius $r_{n} \in(0,+\infty)$, such that onto their boundaries there are no singularities of the function $g(w, i y)$. Hence, by the 
fundamental Cauchy theorem on residues, the sequence of the partial sums can be formed as

$$
\sum_{k=1}^{n} A_{k}(i y)=\frac{1}{2 \pi i} \int_{\Omega_{r_{n}}}^{\cup} g(w, i y) d w
$$

where $A_{k}(i y)=\operatorname{Res}_{w=a_{k}} g(w, i y)$.

On the one hand, on the basis of the second Jordan lemma-[8, Theorem 2, Subsection 3.1.4, Chapter 3, page 52]-if for every $w \in C^{1}$ there exists a unique limiting value $\lim _{|w| \rightarrow+\infty}[w g(w, i y)]=-(1 / 2) f(i y)$, then the sequence of the partial sums $\sum_{k=1}^{n} A_{k}(i y)$ converges, in other words, there exists a sum of the infinite functional series $\sum_{k=1}^{+\infty} A_{k}(i y)$ in the Cauchy sense:

$$
\lim _{n \rightarrow+\infty} \sum_{k=1}^{n} A_{k}(i y)=\operatorname{vp} \sum_{k=1}^{+\infty} A_{k}(i y)=-\operatorname{Res}_{|s|=+\infty} g(w, i y)=-\frac{1}{2} f(i y),
$$

since

$$
\begin{aligned}
\operatorname{Res}_{|w|=+\infty} g(w, i y) & =-\frac{1}{2 \pi i} \lim _{r_{n \rightarrow+\infty}} \int_{\Omega_{r_{n}}}^{\cup} g(w, i y) d w \\
& =-\lim _{|w| \rightarrow+\infty}[w g(w, i y)]=\frac{1}{2} f(i y) .
\end{aligned}
$$

However, on the other hand, by the same Jordan lemma, if $\lim _{|w| \rightarrow+\infty}[w g(w, i y)]$ does not exist for every $w \in C^{1}$, already there exist only partial limiting values:

$$
\lim _{|w| \rightarrow+\infty}[w g(w, i y)]= \begin{cases}-f(i y), & \operatorname{Im} w>0 \\ 0, & \operatorname{Im} w<0\end{cases}
$$

then instead of the limiting value of the sequence of the partial sums $\sum_{k=1}^{n} A_{k}(i y)$ (the Cauchy principal value vp of the infinite series), which does not exist in this acute case, there exists a total value vt of the infinite sum of the residues of the function $g(w, i y)$ that is equal to the limiting sum of the integral values:

$$
\mathrm{vt} \sum_{k=1}^{+\infty} A_{k}(i y)=\frac{1}{2 \pi i} \lim _{r_{n} \rightarrow+\infty}\left[\int_{\Omega_{r_{n}}^{U}}^{n} g(w, i y) d w+\int_{\Omega_{r_{n}}^{L}}^{n} g(w, i y) d w\right],
$$

where the integral paths $\Omega_{r_{n}}^{U}=\left\{w: w(\theta)=r_{n} e^{i \theta} ; \theta \in\left[\delta\left(r_{n}\right), \pi-\delta\left(r_{n}\right)\right]\right\}$ and $\Omega_{r_{n}}^{L}=$ $\left\{w: w(\theta)=r_{n} e^{i \theta} ; \theta \in\left[-\pi+\delta\left(r_{n}\right),-\delta\left(r_{n}\right)\right]\right\}$, are arc of the circular path of integration $\Omega_{r_{n}}$ in the upper $\operatorname{Im} w>0$ and lower $\operatorname{Im} w<0$ half-plane, respectively; and an arbitrary angular function $\delta\left(r_{n}\right)$, which is of sufficiently small real positive values for any positive values of $r_{n}$, satisfies the condition, $\lim _{r_{n} \rightarrow+\infty} \delta\left(r_{n}\right)=0$. In other words, although in this emphasized case there exists no sum of the infinite functional series $\sum_{k=1}^{+\infty} A_{k}(i y)$ in the Cauchy sense, this infinite functional series is summable, that is,

$$
\mathrm{vt} \sum_{k=1}^{+\infty} A_{k}(i y)=-\operatorname{Res}_{|w|=+\infty} g(w, i y)=-\frac{1}{2} f(i y),
$$


since

$$
\begin{aligned}
\operatorname{Res}_{|w|=+\infty} g(w, i y) & =-\frac{1}{2 \pi i} \lim _{r_{n} \rightarrow+\infty}\left[\int_{\Omega_{r_{n}}^{U}}^{n} g(w, i y) d w+\int_{\Omega_{r_{n}}^{L}}^{n} g(w, i y) d w\right] \\
& =\frac{1}{2} f(i y) .
\end{aligned}
$$

Proof. The proof of Theorem 2.1 is almost the very same as that of the ordinary Dirichlet theorem via the calculus of residues, which can be found in many textbooks (the interested reader should consult [8, pages 91-95]), of course in addition to the above mentioned differences. In what follows, without loss of rigorousness, a very brief account will be given of the proof. The procedure is as follows: let the complexvalued function $i y \rightarrow F(i y)$ of the real variable $y$ satisfies the condition of the theorem. Then, the complex-valued function of the complex variables $w$ and $i y$,

$$
g(w, i y)=\frac{1}{1-e^{2 \pi i w}} \mathrm{vt} \int_{-i \pi}^{i y} F(s) e^{w(i y-s)} d s,
$$

(where $i y$ is a point from the interval $(-i \pi, i \pi)$ at which $F(i y)$ has no infinite discontinuity) is an analytic function with respect to $w$, whose infinite discontinuities (singularities) $w_{k}$ are zeros of the function $1-e^{2 \pi i w}, w_{k}= \pm k$ ( $k$ belongs to the set of integers $\mathbb{Z})$. In those circumstances, since for $\operatorname{Im} w>0$ and $i y \in(-i \pi, i \pi)$ : $\lim _{|w| \rightarrow+\infty}\left(1 /\left(1-e^{2 \pi i w}\right)\right)=1$ and $\lim _{|w| \rightarrow+\infty}\left(e^{w(i y-i \pi)} /\left(1-e^{2 \pi i w}\right)\right)=0$, as well as $\lim _{|w| \rightarrow+\infty}\left(1 /\left(1-e^{-2 \pi i w}\right)\right)=0$, it follows immediately from results (2.21) and (2.48) that

$$
\mathrm{vt} \sum_{k=-\infty}^{+\infty} \frac{e^{k i y}}{2 \pi i} \mathrm{vt} \int_{-i \pi}^{i y} F(s) e^{-k s} d s=\frac{1}{2} \lim _{\varepsilon \rightarrow 0^{+}} F(i y-i \varepsilon) .
$$

Similarly, for the complex-valued function

$$
h(w, i y)=\frac{-e^{2 \pi i w}}{1-e^{2 \pi i w}} \mathrm{vt} \int_{i y}^{i \pi} F(s) e^{w(i y-s)} d s,
$$

the following holds:

$$
\mathrm{vt} \sum_{k=-\infty}^{+\infty} \frac{e^{k i y}}{2 \pi i} \mathrm{vt} \int_{i y}^{i \pi} F(s) e^{-k s} d s=\frac{1}{2} \lim _{\eta \rightarrow 0^{+}} F(i y+i \eta) .
$$

2.4. Examples. As an illustration of the use of result of Theorem 2.1, in the first instance it will be shown that the discrete Fourier transform of a real constant function is the periodic Dirac delta function, of the period $2 \pi i$ with respect to $i y$, defined on the closed interval $(-i \pi, i \pi)$ of the imaginary axis in such a way that $\delta(i y)=$ $\lim _{n \rightarrow+\infty} \delta_{n}(i y)$, where

$$
\delta_{n}(i y)=\frac{n}{2}\left[h\left(i y+\frac{1}{n}\right)-h\left(i y-\frac{1}{n}\right)\right],
$$

and $h$ (iy) denotes Heaviside's unit function. Namely, since for any $k \in \mathbb{Z}$

$$
\mathrm{vt} \int_{-i \pi}^{i \pi} \delta(s) e^{-k s} d s=1
$$


then, for any $i y \in[-i \pi, i \pi]$ and $i y \neq 0$, the following holds:

$$
\mathrm{vt} \sum_{k=-\infty}^{+\infty} e^{i y k}=0
$$

In the second instance, the preceding result is proven again, but now in a different way. Namely, since

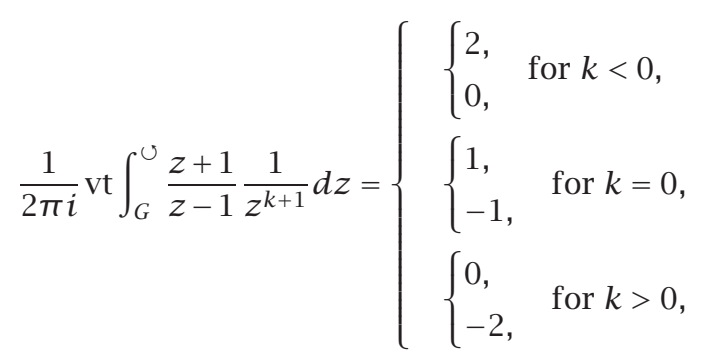

where $G$ is the unit circle in the $z$-plane, then for any $z,|z|=1$ and $z \neq 1$, the following holds:

$$
2 \mathrm{vt} \sum_{k=1}^{+\infty} e^{-i y k}+1=-\frac{i \sin y}{1-\cos y}, \quad-2 \mathrm{vt} \sum_{k=1}^{+\infty} e^{i y k}-1=-\frac{i \sin y}{1-\cos y},
$$

that is,

$$
\mathrm{vt} \sum_{k=1}^{+\infty} \cos (y k)+\frac{1}{2}=0, \quad \mathrm{vt} \sum_{k=1}^{+\infty} \sin (y k)=\frac{1}{2} \frac{\sin y}{1-\cos y},
$$

compare it with [10, page 54].

Moreover, for any $z,|z|>1$ and $|z|<1$,

$$
\operatorname{vp} \sum_{k=1}^{+\infty} z^{-k}=\frac{1}{z-1}, \quad \text { vp } \sum_{k=1}^{+\infty} z^{k}=\frac{z}{1-z}
$$

respectively, since

$$
\frac{1}{2 \pi i} \int_{G_{c}}^{\cup} \frac{z+1}{z-1} \frac{1}{z^{k+1}} d z= \begin{cases}2, & \text { for } k<0 \\ 1, & \text { for } k=0, \\ 0, & \text { for } k>0,\end{cases}
$$

where $G_{c}=\{z:|z|=c, c \in(1,+\infty)\}$, that is,

$$
\frac{1}{2 \pi i} \int_{G_{a}}^{\cup} \frac{z+1}{z-1} \frac{1}{z^{k+1}} d z= \begin{cases}0, & \text { for } k<0 \\ -1, & \text { for } k=0 \\ -2, & \text { for } k>0\end{cases}
$$

where $G_{a}=\{z:|z|=a, a \in(0,1)\}$. 
Finally, in the case of the complex-valued function $z \mapsto z(1-z)^{-2}$, since

$$
\frac{1}{2 \pi i} \mathrm{vt} \int_{G}^{\cup} \frac{1}{(1-z)^{2}} \frac{1}{z^{k}} d z=\left\{\begin{array}{l} 
\begin{cases}k, & \text { for } k<0, \\
0, & \end{cases} \\
0, \text { for } k=0, \\
\begin{cases}0, & \text { for } k>0, \\
k, & \end{cases}
\end{array}\right.
$$

where $G$ is the unit circle in the $z$-plane, for any $z,|z|=1$ and $z \neq 1$, the following holds:

$$
2 \mathrm{vt} \sum_{k=1}^{+\infty} k e^{ \pm i y k}=-\frac{1}{1-\cos y},
$$

that is, for any $z,|z|>1$ and $|z|<1$,

$$
\operatorname{vp} \sum_{k=1}^{+\infty} k z^{-k}=\frac{z}{(z-1)^{2}}, \quad \operatorname{vp} \sum_{k=1}^{+\infty} k z^{k}=\frac{z}{(1-z)^{2}},
$$

respectively.

3. Conclusion. Taking into consideration the fact that the obtained results are theoretical news, we can say that they open up certain possibilities for expansion of the control systems theory. Thus, from the viewpoint of the results, derived in the Subsection 2.2 of this paper for instance, and having in mind the fact that causality related to the area of an expansion of function into Fourier trigonometric series is the theory of the discrete Fourier transform, as well as the theory of the finite and infinite Laplace transform, as it has been shown in this subsection, it is obvious which possibilities are being opened up in the area of control system stability.

On the other hand, disregarding the fact that the results of the paper are, in a certain sense, the theoretical news, some of them have been predictable. So, it is well known that the alternative numerical series $\sum_{k=0}^{+\infty}(-1)^{k}$ has the defined sum, more exactly it is summable and its sum is, by definition, equal to $1 / 2$, just as it has been assumed yet by Euler and Leibniz. Making use of this assumption, they obtained absolutely exact results. There is nothing to be left than to prove exactly the validity of this assumption, as it has been made by result (2.56), that is, (2.59). As for result (2.64), we can say that it is causality related to result (2.56). Namely, since $\sum_{k=1}^{+\infty} k \sin (k t)=0$ for $t=\pi / 2$, that is, $\sum_{k=0}^{+\infty}(2 k+1)(-1)^{k}=0$, it follows that $\sum_{k=0}^{+\infty} 2 k(-1)^{k}=-\sum_{k=0}^{+\infty}(-1)^{k}=-1 / 2$.

\section{REFERENCES}

[1] J. T. Baskin, Some general theorems for evaluating definite integrals by Cauchy's residue theorem, Master's thesis, The Pennsylvania State University, USA, December 1962.

[2] D. L. Debeljkovic, M. B. Jovanovic, S. A. Milinkovic, and L. A. Jacic, Discrete Singular Control Systems, GIP-Culture, Belgrade, 1998 (Serbian).

[3] L. T. Grujic, Discrete Systems, Faculty of Mechanical Engineering, University of Belgrade, Belgrade, 1980 (Serbian).

[4] F. L. Lewis and B. G. Mertzios, On the analysis of discrete linear time-invariant singular systems, IEEE Trans. Automat. Control 35 (1990), no. 4, 506-511. 
[5] B. G. Mertzios and F. L. Lewis, Fundamental matrix of discrete singular systems, Circuits Systems Signal Process. 8 (1989), no. 3, 341-355.

[6] D. S. Mitrinović, Calculus of Residues, Tutorial Text, no. 4, P. Noordhoff Ltd., Groningen, 1966.

[7] _ Classes on Series, Civil book, Belgrade, 1980 (Serbian).

[8] D. S. Mitrinović and J. D. Kečkić, Cauchyjev račun ostataka sa primenama [Cauchy's Calculus of Residues with Applications], Matematički Problemi i Ekspozicije, vol. 8, Naučna Kniga, Belgrade, 1978 (Serbo-Croatian).

[9] __ Mathematics 2, Civil book, Belgrade, 1981 (Serbian).

[10] D. V. Slavić, On summation of series, Univ. Beograd. Publ. Elektrotehn. Fak. Ser. Mat. Fiz. (1970), no. 302-319, 53-59.

Branko SARic: The InStitute "Kirilo SAVic,” 11000 Belgrade, V. STEPe 51, Serbia

E-mail address: bsaric@ptt.yu 


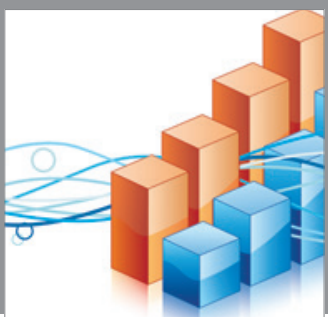

Advances in

Operations Research

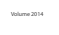

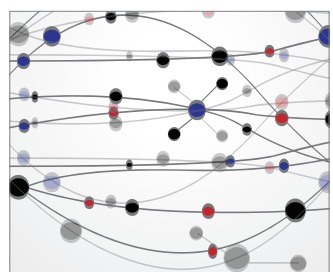

\section{The Scientific} World Journal
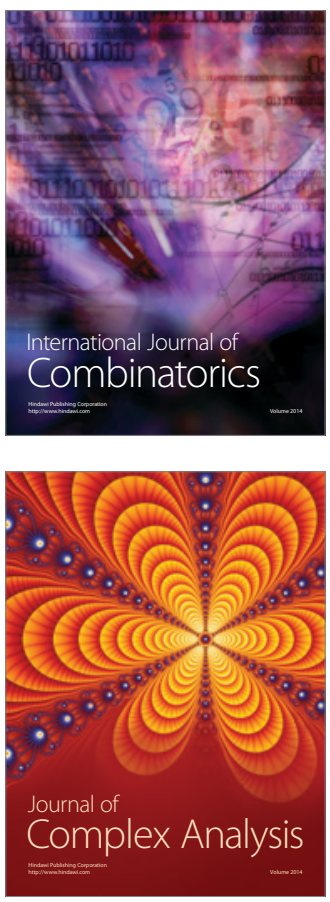

International Journal of

Mathematics and

Mathematical

Sciences
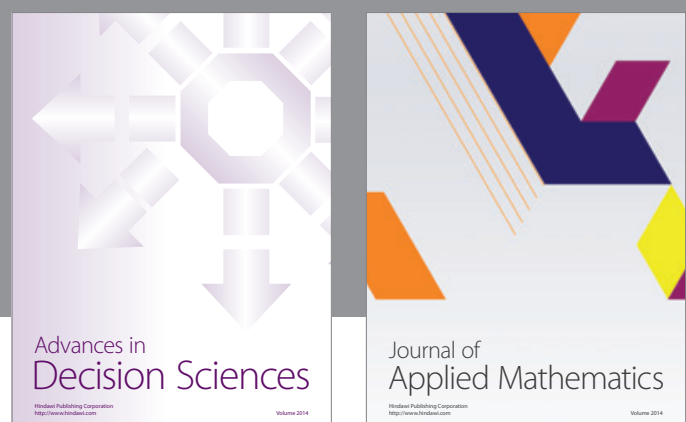

Journal of

Applied Mathematics
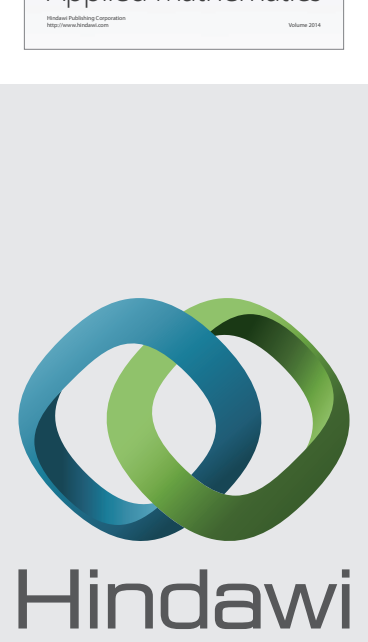

Submit your manuscripts at http://www.hindawi.com
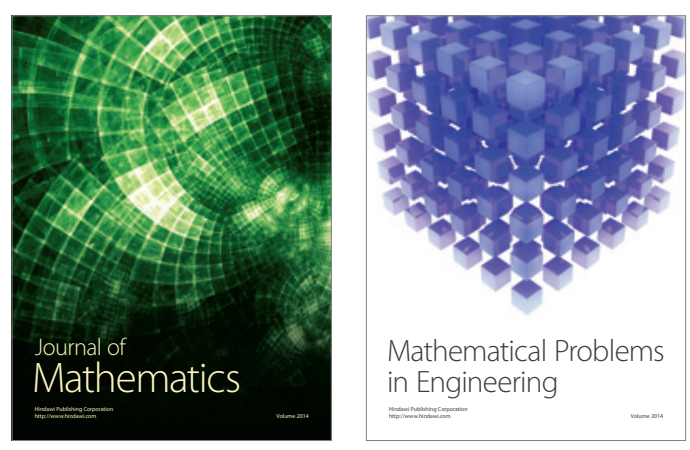

Mathematical Problems in Engineering
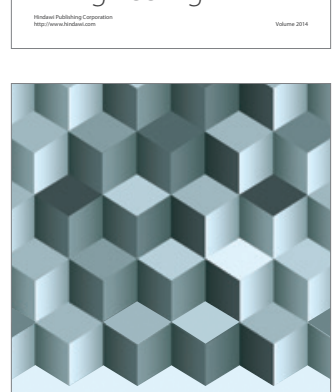

Journal of

Function Spaces
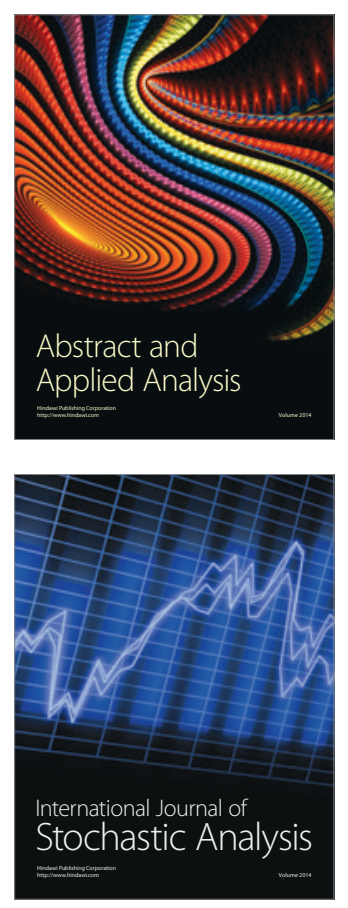

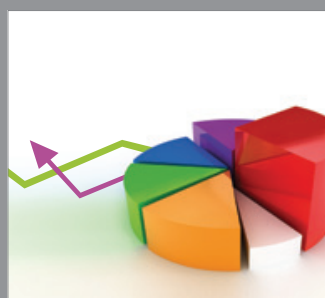

ournal of

Probability and Statistics

Promensencen
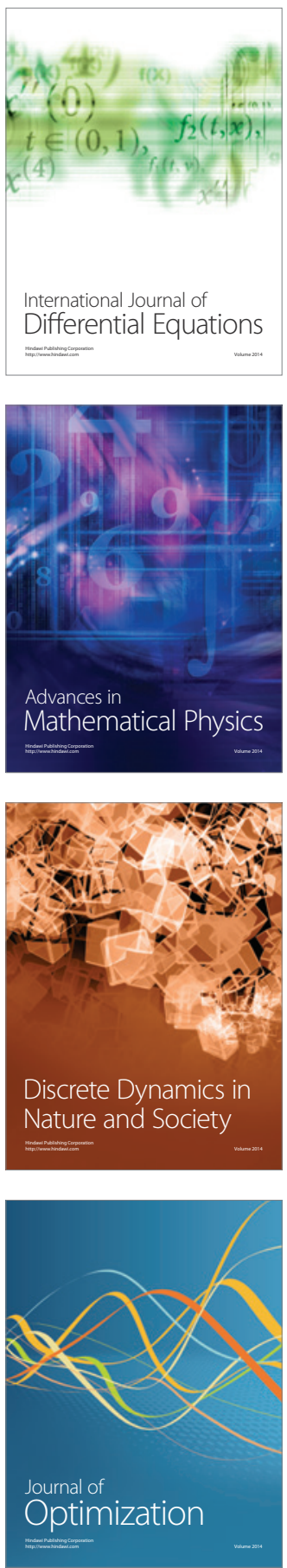